\title{
Harninkontinenz und Descensus Genitalis - Das stille Leiden
}

\author{
D. Fink, Zürich D. Perucchini, Zürich U. Lang, Graz R. Kimmig, Essen
}

\section{Zusammenfassung}

In den letzten Jahren hat sich die Urogynäkologie zu einer sehr innovativen gynäkologischen Subspezialität entwickelt. Neue Erkenntnisse haben zu einer Vielzahl neuer medikamentöser und operativer Therapiemöglichkeiten geführt. Mittels Schlingenoperationen kann in über $80 \%$ der Patientinnen mit einer Belastungsinkontinenz eine Besserung erzielt werden. Ebenso stehen seit kurzer Zeit Medikamente zur Therapie der Belastungsinkontinenz zur Verfügung. Injektionen von Botulinumtoxin $A$ in den Detrusormuskel stellen bei therapieresistenter überaktiver Blase eine Erfolg versprechende Behandlungsstrategie dar.

\section{Urinary Incontinence and Descensus Genitalis - The Silent Sickness}

In the last few years, urogynaecology has become a very innovative gynaecological subspecialty. New findings have led to a multitude of different medicinal and operative therapy options. Stress incontinence can be improved by sling procedures in more than $80 \%$ of patients. Likewise, new drugs to treat stress incontinence have been available for some time. Injections of botulinum toxin $\mathrm{A}$ into the detrusor muscle are a promising treatment strategy in cases of therapy-resistant overactive bladder.

Copyright () 2006 S. Karger AG, Basel

Noch vor wenigen Jahren gehörten unwillkürlicher Urinverlust und Senkungsbeschwerden der Genitalorgane zu Tabuthemen, über die betroffene Frauen häufig nicht einmal mit ihrem Haus- oder Frauenarzt sprachen. Die Annahme, es handle sich dabei um einen unvermeidbaren Preis für das Kinderkriegen und/oder ein unabänderliches Begleitphänomen des Älterwerdens, ist weit verbreitet und hält sich teilweise bis heute. Harninkonti- nenz schränkt die Lebensqualität von Frauen jeglichen Alters jedoch erheblich ein - im Alltag, im Kontakt mit der Familie und mit Freunden, beim Reisen und bei sportlicher Tätigkeit. Die Angst vor unangenehmem Uringeruch und vor sichtbaren Urinflecken hemmt oft die Betroffenen, aus dem Hause zu gehen. Jede dritte Frau gibt nach einer Geburt Inkontinenzprobleme an, und schätzungsweise die Hälfte der Frauen über 50 Jahre zeigt Inkontinenzsymptome. Nur 25\% der betroffenen Frauen haben aber je mit einem Arzt über ihre Beschwerden gesprochen. Bei der Harninkontinenz der Frau kann man deshalb von einem stillen Leiden, ja von einer stillen Epidemie sprechen. Die sich verändernde Altersstruktur der Bevölkerung verbunden mit einem gestiegenen Aktivitätsbedürfnis verschafft der Abklärung und Therapie der Harninkontinenz eine zunehmende Bedeutung. In der öffentlichen Wahrnehmung hat erfreulicherweise auf diesem Gebiet eine Veränderung stattgefunden. Die Betroffenen werden ermutigt, sich durch die Laienpresse und das Internet über Möglichkeiten der Therapie zu informieren.

Vor konservativer oder operativer Therapie bei Harninkontinenz kommt der Diagnostik eine entscheidende Bedeutung zu, da nur wenn diese korrekt durchgeführt wurde, die Therapie erfolgreich sein kann. Gleichzeitig ist eine zielgerichtete Planung der Diagnostik - wie sie in einer modernen urodynamischen Spezialsprechstunde durchgeführt wird - wichtig, um der Patientin unangenehme Untersuchungen zu ersparen, Kosten auf das Nötige zu begrenzen und die Patientin individuell beraten zu können.

In den letzten Jahren haben neue pathophysiologische Erkenntnisse bei Harninkontinenz und bei Senkungserscheinungen zu einer Vielzahl neuer Therapiemöglich-

\section{KARGER \\ Fax +4161306 1234 \\ E-Mail karger@karger.ch}

www.karger.com
(ㄷ) 2006 S. Karger AG, Basel

www.karger.com/ggr
Prof. Daniel Fink

Klinik für Gynäkologie, Departement Frauenheilkunde, Universitätsspital Zürich

Frauenklinikstrasse 10

CH-8091 Zürich (Schweiz)

Tel. +41 4425552 00, Fax +41 4425544 33, E-Mail daniel.fink@usz.ch 
keiten geführt. In der Therapie der Harninkontinenz kam es zu einer rasanten Weiterentwicklung neuer Operationsverfahren. In erfahrenen Händen kann heute mit einem minimal invasiven Eingriff durch Einlage eines suburethral platzierten Bändchens die Belastungsinkontinenz in bis zu 90\% der Fälle behoben werden. Die Operation wird in Lokalanästhesie durchgeführt und erfordert lediglich eine Kurzhospitalisation. Periurethrale submuköse Injektionstechniken («bulking agents») können in ausgewählten Fällen eine Alternative bieten. Injektionen von Botulinumtoxin A in den Detrusormuskel stellen bei therapieresistenter überaktiver Blase eine Erfolg versprechende Behandlungsstrategie dar. Zusätzlich zum empfohlenen Beckenbodentraining stehen neue Medikamente zur Behandlung der Belastungsinkontinenz zur Verfügung.
Ebenso ist die operative Therapie des Descensus genitalis eine der am häufigsten durchgeführten gynäkologischen Operationen. Mit Zunahme des Alters der Bevölkerung wird die Zahl der Frauen, die unter einem Descensus genitalis leiden, ebenfalls steigen. Ziel der chirurgischen Versorgung ist die Beseitigung der klinischen Beschwerden der Patientin, basierend auf einer Wiederherstellung der normalen Anatomie und Funktion. Auf diesem Gebiet hat unter anderem die Einführung der Mesh-Chirurgie die Senkungsoperationen verändert.

In den letzten Jahren hat sich die Urogynäkologie zu einer sehr innovativen gynäkologischen Subspezialität entwickelt. In den USA gibt es in der gynäkologischen Facharztausbildung bereits den entsprechenden Schwerpunktstitel, und in der Schweiz ist ein derartiger in Planung. 